\title{
常绿阔叶林林冠环境对栲幼苗建成的影响
}

\author{
吴小琪 杨圣贺 黄 力 李笑寒 杨 超 钱深华 杨永川 ${ }^{*}$
}

重庆大学三峡库区生态环境教育部重点实验室, 重庆 400045

摘 要 植物幼苗建成阶段是决定种群自然更新的关键生活史阶段。研究林冠环境对常绿阔叶林优势种幼苗建成阶段的影响 对该类森林的恢复和管理具有重要意义。2014-2016年, 该研究在重庆市缙云山国家级自然保护区的常绿阔叶林的不同林冠 环境(大林窗: $>150 \mathrm{~m}^{2}$, 中林窗: 100-150 $\mathrm{m}^{2}$, 小林窗: 50-100 $\mathrm{m}^{2}$, 对照: 林下)下进行栲(Castanopsis fargesii)种子野外播种实 验, 并对栲幼苗命运和生长情况进行了 3年的持续监测。结果表明: (1)栲幼苗出土时间从7月持续到12月, 出苗时间较长, 大林 窗对幼苗出土具有延迟作用; (2)栲种子野外平均萌发率为 $(62.8 \pm 2.0) \%$, 第3个生长季(2016年)末幼苗平均存活率为(65.1 \pm $2.2) \%$, 枯萎是栲幼苗死亡的主要原因; (3)林冠环境对栲种子萌发率及第1个生长季(2014年)末的幼苗存活率无显著影响, 对 第2个(2015年)和第3个生长季末的幼苗存活率具有显著影响; (4)林冠环境在第1个生长季对幼苗生长无明显影响, 但在第2个 和第 3 个生长季具有显著影响, 大、中林窗中幼苗总生物量、株高、基径、根长和叶片数显著高于林下, 比叶面积显著低于林 下; (5) 3 个生长季内, 4类林冠条件下栲幼苗的叶质量比和茎质量比升高, 根质量比和根冠比降低, 并且从第 2 个生长季开始大 林窗中栲幼苗的叶质量比显著高于林下, 根质量比和根冠比显著低于林下。栲幼苗早期的存活和生长依赖种子储存的能量, 受林冠条件影响较弱, 后期则依赖光合作用, 受林冠条件影响较强, 从整个幼苗建成过程看, 大、中林窗更有利于栲幼苗定 居。

关键词 林冠环境; 种子萌发; 幼苗建成; 生长; 栲

吴小琪, 杨圣贺, 黄力, 李笑寒, 杨超, 钱深华, 杨永川 (2019). 常绿阔叶林林冠环境对栲幼苗建成的影响. 植物生态学报, 43, 55-64. DOI: $10.17521 /$ cjpe.2018.0275

\section{Effects of forest canopy condition on the establishment of Castanopsis fargesii seedlings in a subtropical evergreen broad-leaved forest}

WU Xiao-Qi, YANG Sheng-He, HUANG Li, LI Xiao-Han, YANG Chao, QIAN Shen-Hua, and YANG Yong-Chuan* Key Lab of Three Gorges Reservoir Region's Eco-Environment, Ministry of Education, Chongqing University, Chongqing 400045, China

\begin{abstract}
Aims The establishment of seedlings is a key life-history stage during the regeneration of plant populations. This study is to investigate the effects of forest canopy condition (large-sized gap: $>150 \mathrm{~m}^{2}$, medium-sized gap: 100-150 $\mathrm{m}^{2}$, small-sized gap: $50-100 \mathrm{~m}^{2}$, and understory as control) on the establishment of Castanopsis fargesii seedlings, and to guide in situ management and restoration of subtropical evergreen broad-leaved forests.

Methods We conducted a 3-year in situ seed sowing experiment in the Jinyun Mountain National Nature Reserve under different canopy conditions, and monitored the growth and survival of C. fargesii seedlings for three years (2014-2016).

Important findings We found that: (1) the emergence of the seedlings lasted from July to December, and the emergence of seedlings was delayed under large canopy gaps; (2) overall germination rate was $(62.8 \pm 2.0) \%$. The averaged survival rate during three growing seasons was $(65.1 \pm 2.2) \%$, with wither being the key type of seedling mortality; (3) there was no significant difference in the germination and survival rates under different canopy conditions in 2014, but significant difference in the survival rates in 2015 and 2016; (4) seedling growth among canopy conditions showed no difference in 2014, but distinct differences in 2015 and 2016. The seedling growth in large- and medium-sized gaps was significantly promoted compared with those growing under closed canopies in terms of total biomass, plant height, base diameter, root length and leaf number (except for the specific leaf area);

收稿日期Received: 2018-11-03 接受日期Accepted: 2019-01-10

基金项目：国家科技基础性工作专项专题(2015FY210200-16)、重庆市技术创新与应用示范专项重点研发项目(cstc2018jszx-zdyfxmX0007)和重庆市 自然科学基金(CSTC2016jcyjA0379)。Supported by the National Research Basic Work Special Project (2015FY210200-16), Chongqing Technology Innovation and Application Demonstration Major Theme Special Project (cstc2018jszx-zdyfxmX0007), and the Natural Science Foundation of Chongqing (CSTC2016jcyjA0379).

* 通信作者Corresponding author (ycyang@cqu.edu.cn)
\end{abstract}


(5) for each of the four types of canopy conditions, leaf mass ratio (LMR) and stem mass ratio (SMR) of seedlings growing increased within last three years, while root mass ratio $(R M R)$ and root shoot ratio $(R S R)$ decreased. The $L M R$ in large-sized gaps was significantly higher than those in closed canopies, but not for the RMR and $R S R$. The results suggested that the initial survival and growth of $C$. fargesii seedlings may be supported by the nutrients stored in seeds, and thus less affected by canopy conditions. However, in late-establishment stages (i.e., the second and third years), gap size could become a key factor in affecting both the seedling survival and growth. And large- and medium-sized gaps could promote the seedling establishment success for C. fargesii.

Key words canopy condition; seed germination; seedling establishment; growth; Castanopsis fargesii

Wu XQ, Yang SH, Huang L, Li XH, Yang C, Qian SH, Yang YC (2019). Effects of forest canopy condition on the establishment of Castanopsis fargesii seedlings in a subtropical evergreen broad-leaved forest. Chinese Journal of Plant Ecology, 43, 55-64. DOI: $10.17521 /$ cjpe.2018.0275

植物幼苗建成阶段包括种子萌发、出苗、幼苗 存活和生长等过程，该阶段是植物种群自然更新的 关键生活史阶段(Wang \& Smith, 2002; Yang et al., 2015), 并决定着群落物种组成和相对多度(Larson et al., 2015)。深入了解植物种群幼苗增补过程中种 子和幼苗命运的影响因素和异质生境中幼苗生长和 生物量分配策略, 一直以来是植物种群和群落生态 学研究的重点和热点, 具有重要的理论和实践意义 (Yang et al., 2015; Larson et al., 2016; Piiroinen et al., 2017)。

冠层干扰是驱动森林中物种自然更新的一个决 定性因素。冠层干扰所导致的林冠环境的差异直接 影响着其下生境的环境条件(比如光照强度、土壤温 湿度、土壤养分、地表稳定性、调落物厚度、病原 微生物)、资源组合方式以及资源的有效性(Zhu et al., 2003; 欧建德等, 2016; Chmura et al., 2017; Reddy et al., 2017; 董慧和杨立学, 2018), 从而对植 物幼苗建成过程产生影响 (Zhu et al., 2003, 2012; Zhang et al., 2017)。相对而言, 林冠环境对植物种 子萌发阶段, 包括种子萌发率、萌发时间和萌发进 程影响的相关研究较少, 尽管该阶段对后续幼苗命 运、生长以及资源竞争都具有重要的间接作用(Donohue et al., 2010)。相比种子萌发阶段, 木本植物在 幼苗阶段受到的林冠环境影响更强, 相关研究也相 对广泛(Figueroa \& Lusk, 2001)。林冠环境对植物幼 苗的作用主要表现为对其存活率、生物量积累和生 物量分配策略产生影响, 但对不同植物物种影响程 度和方式有所差异。比如林冠干扰引起的光环境的 差异会影响油松(Pinus tabuliformis)、栓皮栎(Quercus variabilis)、岷江冷杉(Abies fargesii var. faxoniana)和小红栲(Castanopsis carlesii)等物种幼苗的存 活率和生长(冯大兰等, 2013; 马莉薇等, 2013; 陈国
鹏等, 2016; 邢忠利等, 2017), 并且可使番龙眼 (Pometia pinnata)、紫楠(Phoebe sheareri)和木荷(Schima superba)等幼苗生物量分配发生变化(汤景明和 翟明普, 2006; 间兴富和曹敏, 2008; 周俊宏等, 2015)。 尽管如此, 目前对植物幼苗建成过程中生物量累积 动态和生物量分配方式出现分化的时间的研究总体 较少, 尤其对具有一定耐阴性质的演替后期种。

亚热带常绿阔叶林生物多样性丰富, 是仅次于 热带雨林的一种具有大生物量和高生产力的生态系 统, 在保护区域生态环境、实现人类可持续发展等 方面均具有十分重要的生态价值(宋永昌, 2013)。亚 热带常绿阔叶林的建群种多为具有一定耐阴性质的 演替后期种, 比如栲(Castanopsis fargesii)、润楠 (Machilus nanmu)和短刺米槠(Castanopsis carlesii var. spinulosa)等, 该类森林的自然更新依赖于冠层 干扰(巩合德等, 2011; Yang et al., 2015; Jin et al., 2018)。近年来, 随着人类活动的增加, 常绿阔叶林 出现生境破碎化和优势种衰退等问题(宋永昌, 2013), 导致林冠环境的剧烈变化, 该类森林的管理 和恢复日益受到重视, 而其有效性应当建立在对常 绿阔叶林优势种自然更新过程深入认识的基础上。 因此, 研究冠层干扰所导致的环境异质性对常绿阔 叶林优势种建成过程的影响能够为对该类森林的管 理、保护和恢复提供科学参考。

栲是亚热带常绿阔叶林的主要优势种, 广泛分 布于长江以南地区, 在区域生态系统中发挥着重要 作用。近年来, 栲面临严重的衰退, 如在缙云山, 记 录到 $84.5 \%$ 的大径级个体存在衰退症状(高祥阳, 2015)。栲生活史的解析是衰退栲林管理与修复的科 学基础, 而这一长寿命树种生活史早期的研究在种 子萌发和当年生幼苗的生长方面有少量探索, 但缺 乏相对长期的原位监测, 对幼苗命运的分析主要基 
于空间代替时间进行, 缺乏生态过程的研究, 具有 很大的不确定性(Yang et al., 2015)。本研究以缙云山 亚热带常绿阔叶林主要优势种栲为研究对象, 在林 冠干扰形成的不同大小林窗和林下进行野外播种实 验, 并对萌发幼苗进行了为期 3 年的持续监测, 探讨 林冠环境对栲种子萌发、幼苗存活和幼苗生物量积 累与分配的影响, 揭示常绿阔叶林优势种幼苗建成 过程中对异质生境的适应机制, 以期为受损常绿阔 叶林的恢复提供理论参考。

\section{1 研究方法}

\section{1 研究区概况}

研究地位于重庆市北碚区缙云山国家级自然保 护区 $\left(29.81^{\circ}-29.86^{\circ} \mathrm{N}, 106.34^{\circ}-106.41^{\circ} \mathrm{E}\right)$, 区内海 拔180-951.5 m。该区属典型的亚热带季风气候, 年 平均气温 $13.6{ }^{\circ} \mathrm{C}$, 最冷月 $\left(1\right.$ 月)平均气温 $3.1{ }^{\circ} \mathrm{C}$, 最 热月(7月)平均气温 $24.3{ }^{\circ} \mathrm{C}$, 年降水量 $1611.8 \mathrm{~mm}$, 相对湿度 $87 \%$ 。缙云山土壤类型以炭质页岩、石英 砂岩和泥质砂岩为母质风化而成的酸性黄壤为主 (Yang et al., 2015)。缙云山植被为典型的亚热带常绿 阔叶林, 主要优势树种为栲、短刺米槠、润楠、四 川大头茶 (Polyspora speciosa) 和光亮山矾 (Symplocos lucida)等(高祥阳, 2015)。

\section{2 研究物种}

栲是壳斗科雉属(Castanopsis)的高大常绿乔木, 亚热带常绿阔叶林常见优势种, 以栲为建群种的常 绿阔叶林广泛分布于中国的长江流域。栲林多分布 在海拔 $1400 \mathrm{~m}$ 以下的地区, 其分布地受人类活动 影响较大, 目前大面积的完整栲林十分稀少。栲种 子成熟期为每年的 10-12月，掉落高峰期为 10 月下 旬。种子平均鲜质量为 $0.46 \mathrm{~g}$, 室内(培养箱)种子萌 发率为 $75.1 \%$, 种子大小对萌发率和萌发进程具有 影响(黄力, 2016)。栲种子初次散布方式为重力散布, 二次散布方式为动物散布, 啮齿动物是其种子的主 要捕食者和散布者(Yang et al., 2015)。

\section{3 种子收集和保存}

2013年10月下旬开始，从缙云山常绿阔叶林永 久监测样地布设的种子收集框中收集栲种子(黄力, 2016)。在实验室中使用浮水法去除虫蛙、啃食和败 育的种子, 并选取大小一致 $(0.4 \mathrm{~g}$ 左右, 尽量排除种 子大小对野外萌发率和萌发格局产生的潜在影响) 的完好种子, 表面消毒处理后置湿沙保存(高祥阳
等, 2016)。

\section{4 播种样方的选择和播种}

在缙云山青龙寨保存较为完好的常绿阔叶林中 选择坡度、坡向基本一致的林窗，依据面积大小设 置林窗梯度, 即大林窗 $\left(>150 \mathrm{~m}^{2}\right)$ 、中林窗(100-150 $\mathrm{m}^{2}$ ) 和小林窗(50-100 $\left.\mathrm{m}^{2}\right)$, 并以林下作为对照, 共4 个处理, 构成林冠梯度。每个处理设置 3 个重复( $\mathrm{Zhu}$ et al., 2003), 不同林冠环境下的光通量存在显著差 异(平均值土标准误差, 样方详细信息见表1)。

2014年3月初进行野外播种, 清除播种样方中 土壤表层自然散落的栲种子、凋落物和植物个体, 并且对新生植物个体做定期的清除。在每个样方中 设置9个播种样格 $(1 \mathrm{~m} \times 0.5 \mathrm{~m})$ 。在每个播种样格土 壤表层(深0.5 cm)均匀埋藏15粒种子(参照2013年栲 成熟完好种子的平均种子雨密度), 种子脐部朝上。 每个样方播种135粒, 总计 1620 粒。播种完成后, 覆 盖上铁丝网罩 (孔径 $1.4 \mathrm{~cm} \times 1.4 \mathrm{~cm})$, 以避免啮齿动 物对种子的啃食或搬运(杜彦君等, 2007)。

\section{5 种子萌发、出苗及幼苗命运监测}

2014年3月起, 每个月末对播种样方中的种子 萌发情况进行仔细观察, 标记萌发个体, 并计数, 至连续两次未发现新个体结束。并于2014-2016年每 年12月底, 对幼苗命运进行监测, 统计存活幼苗数, 并记录幼苗的死亡原因, 分为枯死、物理损伤、动 物啃食和原因不明四个类型。

\section{6 幼苗性状测定}

分别于2014、2015和2016年12月底，即每个生 长季末, 在每播种样方中随机选取 3 个播种样格, 将 所有栲幼苗个体全株挖出, 带回实验室后测量幼苗 株高 $(\mathrm{cm})$ 、基径 $(\mathrm{mm}) 、$ 根长 $(\mathrm{cm})$ 、叶片数、叶面积 $\left(\mathrm{cm}^{2}\right)$ 等指标。用流水冲洗幼苗表面泥土, 然后将幼 苗根、茎和叶分离(挖取时已标注幼苗地上与地下部 分分界线), 置烘箱 $105{ }^{\circ} \mathrm{C}$ 杀青 $15 \mathrm{~min}$, 然后 $70{ }^{\circ} \mathrm{C}$ 烘 干 $48 \mathrm{~h}$, 称量幼苗各部分质量, 精确到 $0.001 \mathrm{~g}$ 。

\section{7 数据处理}

种子萌发率、萌发进程、幼苗存活率、比叶面 积 $(S L A)$ 、叶质量比 $(L M R)$ 、茎质量比 $(S M R)$ 、根质 量比 $(R M R)$ 及根冠比 $(R S R)$ 的计算公式如下:

种子萌发率 $(\%)=$ 萌发的种子数/初始种子数 $\times$ $100 \%$

萌发进程 $(\%)=$ 累积萌发的种子数/初始种子数 $\times 100 \%$ 
表1 林窗和林下播种样方基本信息

Table 1 Basic characteristics of sowing plots in forest gaps and understory

\begin{tabular}{|c|c|c|c|c|c|c|c|c|}
\hline \multirow{2}{*}{\multicolumn{2}{|c|}{$\begin{array}{l}\text { 林冠环境 } \\
\text { Canopy condition }\end{array}$}} & \multirow{4}{*}{$\begin{array}{l}\text { 面积 } \\
\text { Area }\left(\mathrm{m}^{2}\right)\end{array}$} & \multirow{4}{*}{$\begin{array}{l}\text { 形成方式 } \\
\text { Types of formation } \\
\text { 人工砍伐栲 } \\
\text { Selective cutting of } \\
\text { Castanopsis fargesii }\end{array}$} & \multirow{4}{*}{$\begin{array}{l}\text { 年龄/年 } \\
\text { Age (year) }\end{array}$} & \multicolumn{3}{|c|}{ 主要边界木 Main boundary trees } & \multirow{4}{*}{$\begin{array}{c}\begin{array}{c}\text { 光通量 } \\
\text { Quantum flux } \\
\left(\mu \mathrm{mmol} \cdot \mathrm{m}^{-2} \cdot \mathrm{s}^{-1}\right)\end{array} \\
484 \pm 48.4^{\mathrm{a}}\end{array}$} \\
\hline & & & & & \multirow{3}{*}{$\begin{array}{l}\text { 物种 Species } \\
\text { 栲 C. fargesii } \\
\text { 山矾 Symplocos sumunti }\end{array}$} & \multirow{2}{*}{$\frac{\text { 胸径 } D B H(\mathrm{~cm})}{20-45}$} & \multirow{2}{*}{$\frac{\text { 树高 } H(\mathrm{~m})}{15-25}$} & \\
\hline 大林窗 ～～～～～～～～～ & A & & & & & & & \\
\hline Large-sized gap & & & & & & $10-20$ & $10-20$ & \\
\hline & B & 185 & 栲倒木 C. fargesii logs & $3-5$ & 栲 C. fargesii & $30-40$ & $15-20$ & $513 \pm 30.9^{\mathrm{a}}$ \\
\hline & & & & & 润楠 Machilus nanmu & $20-35$ & $15-25$ & \\
\hline & $\mathrm{C}$ & 190 & 栲倒木 C. fargesii logs & $6-8$ & 栲 C. fargesii & $25-50$ & $17-20$ & $697 \pm 104.3^{\mathrm{a}}$ \\
\hline & & & & & 润楠 M. nanmu & $20-35$ & $10-20$ & \\
\hline \multirow{7}{*}{$\begin{array}{l}\text { 中林窗 } \\
\text { Medium-sized } \\
\text { gap }\end{array}$} & A & 122 & 栲倒木 C. fargesii logs & $5-7$ & 润楠 M. nanmu & $20-30$ & $15-25$ & $365 \pm 24.4^{\mathrm{b}}$ \\
\hline & & & & & 山矾 S. sumuntia & $10-20$ & $10-20$ & \\
\hline & & & & & $\begin{array}{l}\text { 短刺米槠 Castanopsis carle- } \\
\text { sii var. spinulosa }\end{array}$ & $15-30$ & $10-15$ & \\
\hline & B & 140 & 栲倒木 C. fargesii logs & $5-7$ & 润楠 M. nanmu & $15-30$ & $15-25$ & $447 \pm 36.5^{\mathrm{b}}$ \\
\hline & $\mathrm{C}$ & 131 & 栲倒木 C. fargesii logs & $5-7$ & 栲 C. fargesii & $35-45$ & $15-20$ & $444 \pm 53.9^{b}$ \\
\hline & & & & & 润楠 M. nanmu & $10-20$ & $15-20$ & \\
\hline & & & & & 山矾 S. sumuntia & $10-20$ & $10-20$ & \\
\hline \multirow{8}{*}{$\begin{array}{l}\text { 小林窗 } \\
\text { Small-sized gap }\end{array}$} & A & 88 & 栲倒木 C. fargesii logs & $5-7$ & 栲 C. fargesii & $35-55$ & $20-25$ & $169 \pm 30.7^{\mathrm{c}}$ \\
\hline & & & & & 润楠 M. nanmu & $25-35$ & $20-25$ & \\
\hline & $\mathrm{B}$ & 72 & 栲倒木 C. fargesii logs & $4-6$ & 栲 C. fargesii & $40-50$ & $20-25$ & $191 \pm 13.3^{\mathrm{c}}$ \\
\hline & & & & & 润楠 M. nanmu & $20-30$ & $15-25$ & \\
\hline & $\mathrm{C}$ & 75 & 栲倒木 C. fargesii logs & $5-7$ & 栲 C. fargesii & $25-40$ & $15-20$ & $199 \pm 9.3^{\mathrm{c}}$ \\
\hline & & & & & 润楠 M. nanmu & $10-20$ & $15-20$ & \\
\hline & & & & & 主要优势种 Mai & in dominant species & & \\
\hline & & & & & 物种 Species & 胸径 DBH (cm) & 树高 H (m) & \\
\hline \multirow{5}{*}{$\begin{array}{l}\text { 林下 } \\
\text { Understory }\end{array}$} & A & 100 & - & - & 栲 C. fargesii & $35-55$ & $20-25$ & $40 \pm 4.1^{\mathrm{d}}$ \\
\hline & & & & & 润楠 M. nanmu & $35-45$ & $20-25$ & \\
\hline & B & 100 & - & - & 栲 C. fargesii & $40-50$ & $20-25$ & $35 \pm 3.4^{\mathrm{d}}$ \\
\hline & $\mathrm{C}$ & 100 & - & - & 栲 C. fargesii & $35-45$ & $20-25$ & $39 \pm 3.9^{\mathrm{d}}$ \\
\hline & & & & & 冬桃 Elaeocarpus duclouxii & $20-35$ & $15-25$ & \\
\hline
\end{tabular}

一表示无。不同小写字母表示林冠环境间光通量差异显著 $(p<0.05)$ 。

- means nothing. Different lowercase letters showed significant differences among canopy conditions' quantum flux $(p<0.05)$.

幼苗存活率 $(\%)=$ 幼苗存活数 $/$ 萌发种子数 $\times 100 \%$ 比叶面积 $\left(\mathrm{cm}^{2} \cdot \mathrm{g}^{-1}\right)=$ 新鲜叶的单叶面积/叶干质量 叶质量比 $\left(\mathrm{g} \cdot \mathrm{g}^{-1}\right)=$ 叶生物量/总生物量 茎质量比 $\left(\mathrm{g} \cdot \mathrm{g}^{-1}\right)=$ 茎生物量 $/$ 总生物量 根质量比 $\left(\mathrm{g} \cdot \mathrm{g}^{-1}\right)=$ 根生物量/总生物量

根冠比 $\left(\mathrm{g} \cdot \mathrm{g}^{-1}\right)=$ 地下部分生物量/地上部分生物量 使用单因素方差分析判断林冠环境是否显著影 响种子萌发率及幼苗存活率; 通过多重比较, 了解 林冠梯度间的种子萌发率、幼苗存活率、幼苗生长 指标及生物量分配是否存在显著性差异，显著性水 平为 0.05 。数据分析使用 $\mathrm{R}$ 3.3.1软件完成。

\section{2 结果}

\section{1 栲种子萌发格局}

栲种子整体萌发率为 $(62.8 \pm 2.0) \%$ (平均值土标
准误差，下同)。林冠环境对种子萌发率无显著影响 $(p>0.05)$, 大林窗、中林窗、小林窗和林下环境中 萌发率分别为 $(63.2 \pm 3.5) \% 、(63.2 \pm 3.8) \%$ 、(61.3 \pm $4.5) \%$ 和(64.4 \pm 4.6$) \%$ (图1)。

总体上，栲种子于7月初开始萌发出土，萌发时 间集中在7、8月份，至12月底萌发结束，萌发持续时 间约 5 个月。不同林冠环境下栲种子萌发的时间格局 存在一定差异，林下的栲种子于7月达到萌发高峰， 林窗中的栲种子于 8 月达到萌发高峰期, 林下和中、 小林窗中栲种子萌发进程快于大林窗。此外, 大林 窗中9-11月萌发的栲种子明显多于中林窗和小林窗 (图2)。

\section{2 栲幼苗的存活动态}

2014、2015和2016年生长季末的幼苗平均存活 率分别为 $(89.7 \pm 1.2) \% 、(76.0 \pm 1.9) \%$ 和 $(65.1 \pm$ www.plant-ecology.com 


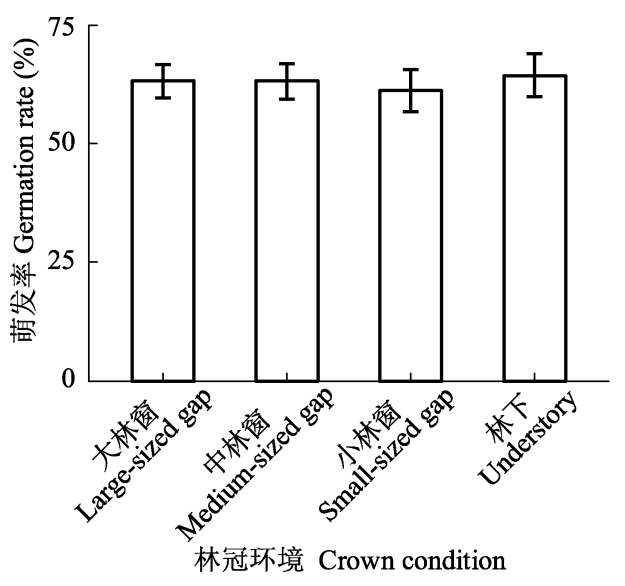

图1 不同林冠环境下的栲种子萌发率(平均值土标准误差)。

Fig. 1 The germination rate of Castanopsis fargesii seeds under different canopy conditions (mean $\pm S E$ ).
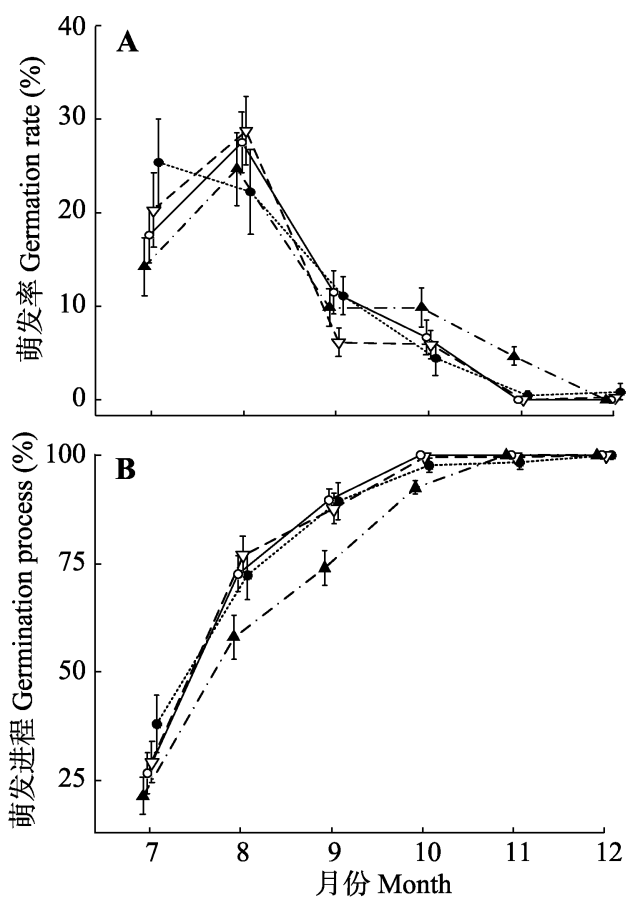

- - 大林窗 Large-sized gap —— 中林窗 Medium-sized gap

$\bullet \bullet-$ 小林窗 Small-sized gap ……... 林下 Understory

图2 不同林冠环境下栲种子的月萌发率和萌发进程(平均 值土标准误差)。

Fig. 2 Monthly germination rate and germination process of Castanopsis fargesii seeds under different canopy conditions (mean $\pm S E)$.

2.2)\%。林冠环境对栲在2014年生长季末的幼苗存活 率无显著影响 $\left(p_{2014}=0.19\right)$, 但对 2015年和2016年 生长季末的幼苗存活率具有显著影响 $\left(p_{2015}=0.0416\right.$, $\left.p_{2016}=2.53 \times 10^{-4}\right)$, 大林窗和中林窗环境下的幼苗 存活率显著高于小林窗和林下(图3)。不同林冠环境 下幼苗死亡原因都以干枯为主, 小林窗和林下物 理损伤死亡个体比例显著高于大林窗和中林窗 (图4)。

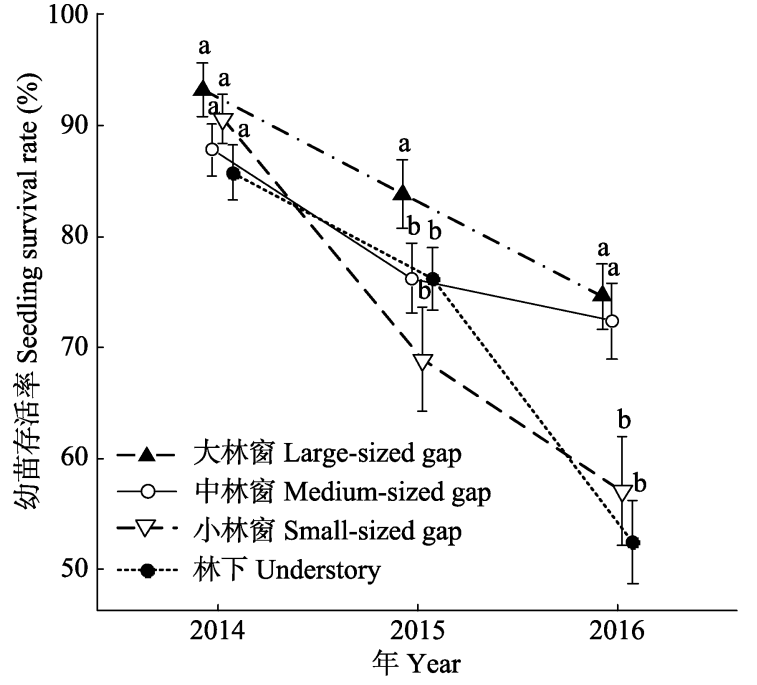

图3 不同林冠环境下栲幼苗存活率的时间动态(平均值 \pm 标 准误差)。不同小写字母表示不同林冠环境间幼苗存活率差 异显著 $(p<0.05)$

Fig. 3 Temporal dynamics of Castanopsis fargesii seedling survival rates (mean $\pm S E$ ) under different canopy conditions. Different lowercase letters showed significant differences among seedling survival rate under different canopy conditions $(p<$ $0.05)$.

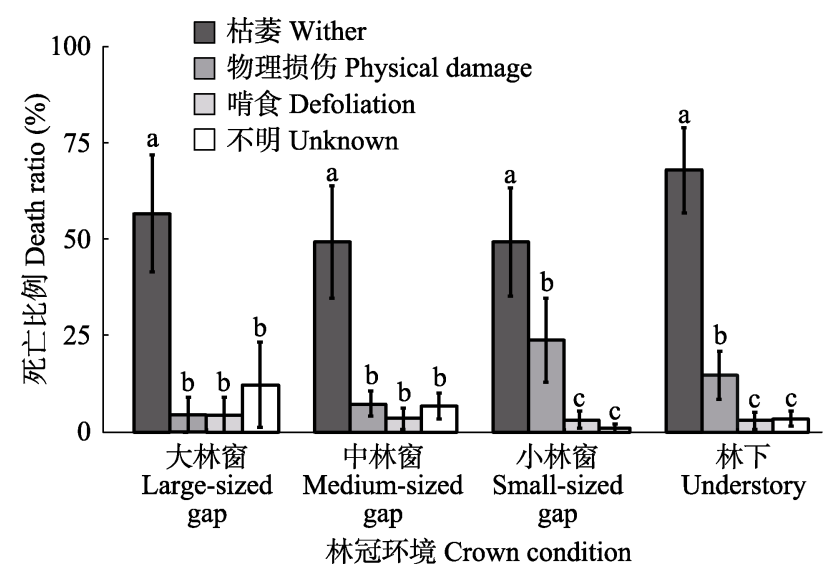

图4 不同林冠环境下栲幼苗死亡原因比例(平均值土标准误 差)。不同小写字母表示该环境下幼苗死亡原因比例间差异 显著 $(p<0.05)$

Fig. 4 Ratio of the causes of Castanopsis fargesii seedling death under different canopy conditions (mean $\pm S E$ ). Different lowercase letters showed significant differences among the causes of seedling death under same canopy condition $(p<0.05)$.

\section{3 幼苗生长和生物量分配}

2014年生长季末, 4种林冠环境下栲幼苗的总生 物量、株高、基径、根长和叶片数无显著差异 $(p>$ 0.05)；2015年和2016年生长季末，4种林冠环境下栲 幼苗的总生物量、株高、基径、根长和叶片数有显 著差异 $(p<0.05)$, 大林窗和中林窗幼苗的生长指标 显著高于林下和小林窗; 3 个生长季末, 林下幼苗的 比叶面积都显著高于林窗 $(p<0.05$, 图5)。 


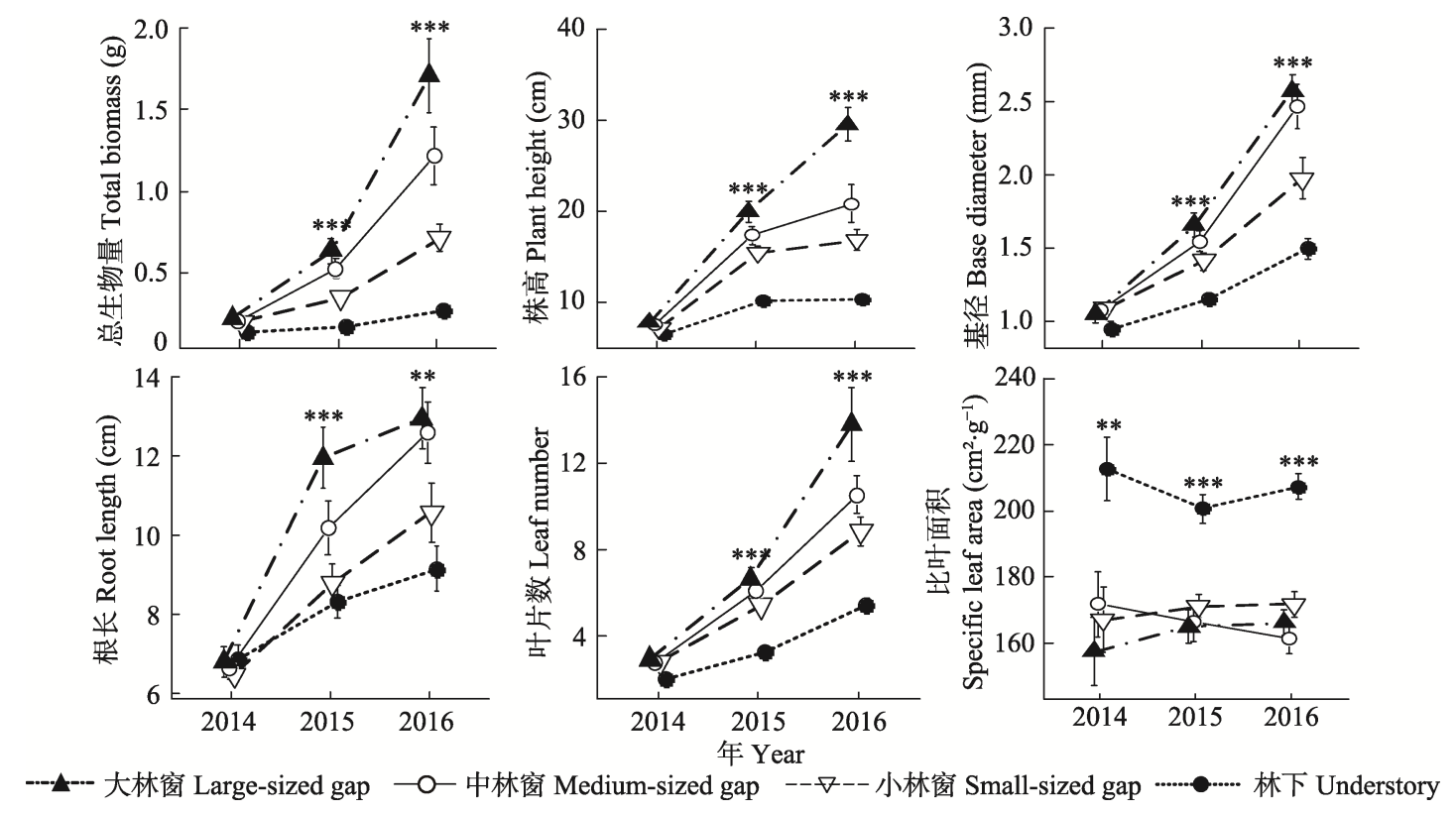

图5 不同林冠环境下栲幼苗生长指标的年际动态(平均值沶准误差)。*表示林冠环境对该阶段幼苗生长指标有着显著影响 $(* *, p<0.01 ; * * *, p<0.001)$ 。

Fig. 5 The interannual variation of the growth indicators for Castanopsis fargesii seedling under different canopy conditions (mean $\pm S E)$. * indicates a significant canopy effect on a specific seedling growth indicator at a given stage $(* *, p<0.01 ; * * *, p<0.001)$.

2014到2016年, 4种林冠环境下栲幼苗叶质量比 和茎质量比随时间增加, 而根质量比和根冠比随时 间降低; 2014年生长季末, 林冠环境对栲幼苗叶质 量比、茎质量比和根质量比都无显著影响 $(p>0.05)$, 但对根冠比有显著影响 $(p<0.05) ; 2015$ 和 2016 年生 长季末, 林冠环境对栲幼苗的叶质量比、根质量比 和根冠比都具有显著影响 $(p<0.05)$, 但对茎质量比 无显著影响 $(p>0.05$, 图6)。

\section{3 讨论}

\section{1 林冠环境对种子萌发格局的影响}

种子萌发是植物离开相对安全的种子阶段进入 高度脆弱的幼苗期的关键过渡时段(Gremer et al., 2016), 林冠干扰所导致的林冠环境差异对植物种 子萌发过程影响的研究相对较少。本研究发现, 栲 种子在野外的平均萌发率为 $(62.8 \pm 2.0) \%$, 略低于 室内萌发率 $(75.1 \%$, 高祥阳等, 2016), 但比壳斗科 的大部分物种萌发率高(Abe et al., 2001; Li \& Ma, 2003; Zheng et al., 2009)。种子的野外萌发率是反映 植物生殖潜力的关键性状, 栲种子较高的野外萌发 率是维持种群自然更新和在群落中优势地位的基 础。此外, 我们发现, 林冠环境对栲种子的萌发率无 显著影响(图2)，与陈波等(2002)的研究结果一致。 栲种子质量较大, 其萌发率主要取决于种子自身营
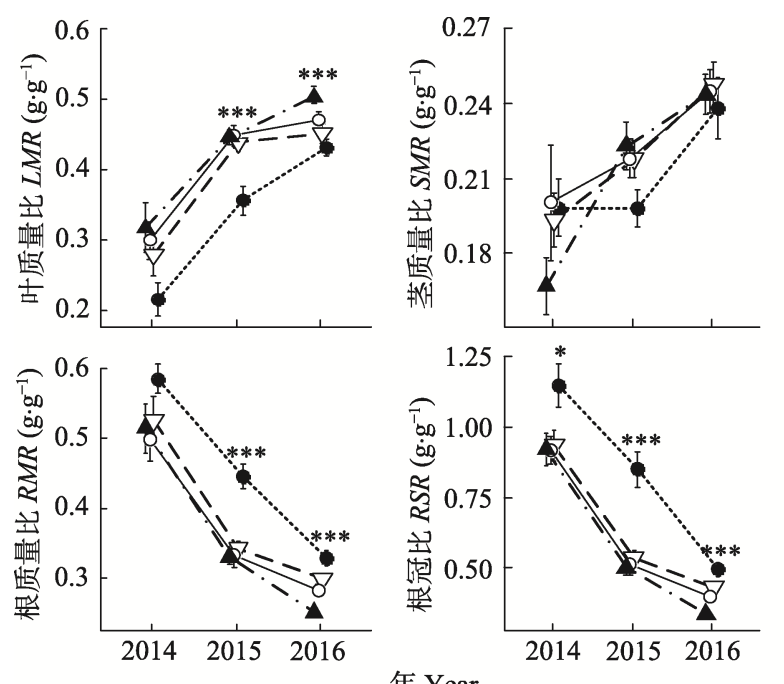

-.-.... 大林窗 Large-sized gap

Year

--マ-- 小林窗 Small-sized gap

一林窗 Medium-sized gap 林下 Understory

图6 不同林冠环境下栲幼苗生物量分配的年际动态(平均 值土标准误差)。*表示林冠环境对该阶段幼苗生物量分配有 着显著影响 $(* *, p<0.01 ; * * *, p<0.001)$ 。

Fig. 6 The interannual variation of the biomass allocation for Castanopsis fargesii seedlings under different canopy conditions (mean $\pm S E$ ). $L M R$, leaf mass ratio; $S M R$, stem mass ratio; $R M R$, root mass ratio; $R S R$, root shoot ratio. * indicates a significant canopy effect on seedling biomass allocation at a given stage $(* *, p<0.01 ; * * *, p<0.001)$.

养物质的含量, 而受光异质性影响较弱(Figueroa \& Lusk, 2001; 陈圣宾等, 2005)。

种子的萌发时间对幼苗存活和生长都具有重要 
作用, 并且易受环境因子影响(Gremer et al., 2016)。 总体上看, 栲种子在野外萌发持续时间较长, 分散 的出苗格局避免了偶然因素对新萌发幼苗带来的灾 难性影响, 可能是栲等演替后期种的种群更新适应 性策略。不同于种子萌发率, 本研究发现林冠环境 对栲种子的萌发时间具有明显影响, 林下萌发最快, 并且大林窗对萌发具有迟滞作用, 与同为壳斗科植 物的辽东栋(Quercus wutaishanica)相似(闰兴富等, 2011)。大林窗中种子的延迟萌发可规避强光照射对 新幼苗产生光伤害, 对幼苗在野外的早期生存具有 积极作用(Zhu et al., 2003)。

\section{2 林冠环境对幼苗存活的影响}

植物幼苗在建成之前十分脆弱, 极易受到环境 因素的影响(Clark et al., 2013), 植物建成过程中幼 苗存活和主要致死机制的研究对预测种群动态和分 布范围具有重要意义。以往针对幼苗建成的研究多 只持续1年(陈波等, 2002; 间兴富等, 2011), 极少持 续 3 年及以上。对于某些种子质量较大的物种, 其幼 苗在第 1 个生长季的存活和生长所需的能量主要来 自种子中咜存的营养物质(李亚男等, 2014), 而异质 环境对幼苗产生作用的时间是在种子能量消耗完之 后, 因此短期的研究可能导致研究结论出现偏差。 本研究对栲幼苗建成过程进行了为期 3 年的追踪调 查, 能够更加全面和深入地揭示环境因子对幼苗存 活的影响。

4种林冠环境下, 栲幼苗在第 1 个生长季的死亡 个体比例都较低(大林窗: $6.8 \%$, 中林窗: $12.2 \%$, 小 林窗: 9.4\%, 林下: 14.3\%), 从第2个生长季(2015年) 开始幼苗死亡加速, 尤其是林下和小林窗。尽管不 同林冠环境下栲幼苗死亡方式具有一定差异, 但枯 萎死亡是最主要的形式。造成植物幼苗枯萎死亡的 主要机制在不同生境中可能存在差异, 比如林窗生 境中幼苗枯死的原因可能与干旱有关(Engelbrecht et al., 2006; Bonal et al., 2016), 林下生境中幼苗枯 萎死亡的原因可能与病原菌侵害造成根系腐烂而失 去对营养物质和水分的吸收有关。林冠环境从第2 个生长季开始对幼苗存活产生明显影响, 第3个生 长季(2016年)结束后, 大林窗和中林窗幼苗存活率 显著高于小林窗和林下(图3)。栲被认为是具有耐阴 性质的演替后期种, 其更新为逐株替代的方式, 幼 苗能够在光照较弱的林下生境中存活较长时间。尽 管如此, 林冠环境的改变对幼苗存活具有较强的促
进作用。

\section{3 林冠环境对幼苗生长和生物量分配的影响}

植物幼苗的高度和生物量大小是决定其能否成 功定居的关键因素(Comita \& Hubbell, 2009; Chanthorn \& Caughlin, 2013; Lu et al., 2015), 生长状况 良好的个体存活概率也较高。研究植物幼苗在异质 生境中的生长, 对了解物种幼苗建成过程中的适应 机制和预测幼苗的存活具有重要意义。本研究发现, 第 2 个生长季末, 大林窗和中林窗幼苗的总生物量、 株高、基径、根长和叶片数等生长指标显著高于小 林窗和林下, 并且大林窗和中林窗中幼苗的相对生 长速率也明显较高(图4)。此结果表明, 从第2个生长 季开始, 林窗对栲幼苗生长具有明显的促进作用, 并且这种促进作用越来越强。同种子萌发相似, 栲 幼苗在生长初期(第 1 个生长季)所需能量主要来自 种子, 因此反映幼苗生长状况的各项指标在不同林 冠环境下未出现明显分化。随着种子能量的消耗, 光合作用所固定的能量成为幼苗生长的主要能量来 源, 小林窗和林下相对较弱的光照使栲幼苗生长受 到抑制。尽管栲具有一定的耐阴性质，但是大林窗 和中林窗中良好的光照条件对其幼苗生长具有显著 的促进作用，并且随着时间延长不断增强。

资源的适应性分配可提高植物幼苗的生存适合 度, 是植物适应异质生境的重要对策(侯兆疆等, 2014)。叶质量比、茎质量比和根质量比反映了植物 在叶、茎和根3种功能器官之间的碳分配格局, 根冠 比代表了植物生物量在地上部分和地下部分之间的 分配情况, 被作为植株对土壤水分、养分和光资源 适应性分配的指标。通常认为, 当某种资源出现限 制时，植株将更多的生物量分配给吸收该类资源的 器官, 以平衡资源限制(Morales et al., 2015)。本研究 发现, 在3个生长季内, 4 种林冠环境下的栲幼苗都 逐渐将更多的能量分配给叶和茎在内的地上部分, 并且大林窗和中林窗中的幼苗分配给地上部分的能 量比例显著大于小林窗和林下的分配比例(图6)。在 小林窗和林下, 由于光照限制, 栲幼苗将更多的生 物量分配给地上部分, 并且通过增大叶片比叶面积 的方式提高光合速率，以适应林下低光环境，栲幼 苗在林下生长缓慢。大林窗和中林窗中, 栲幼苗具 有较高的净光合速率(丁圣彦和卢训令, 2007), 地上 部分生物量累积速度很快, 从而表现为比林下更低 的根冠比和更高的叶质量比。常绿榈叶林中林窗形 
成后郁闭时间较短, 栲幼苗在获得适宜的光环境后 将快速累积的生物量分配给地上部分的叶和茎, 可 获得更多的光资源, 增强与林窗中先锋树种对光资 源的竞争能力, 对促进种群更新具有重要作用。另 外, 不同林冠环境下, 栲幼苗均将较少的能量分配 给地下部分, 表明在常绿阔叶林中, 水分和营养物 质可能不是其幼苗生长的主要限制因子(Tian et al., 2017)。

\section{4 结论和建议}

栲种子野外萌发持续时间较长, 萌发率较高; 幼苗建成初期的存活和生长主要依赖种子内储存的 营养物质, 受林冠环境影响不显著; 随着种子内营 养物质的消耗, 幼苗生长主要依赖光合作用, 林冠 环境对幼苗存活和生长产生显著影响, 大、中林窗 中的幼苗具有更高的存活率, 并且幼苗总生物量、 株高、基径、根长和叶数等生长指标及叶生物量分 配均显著高于林下。综上, 尽管栲具有一定耐阴性, 但林下光环境的改善将促进幼苗建成后期阶段的存 活和生长, 大、中林窗更有利于栲幼苗建成。

常绿阔叶林的多数优势种具有和栲相似的物种 特性, 林窗干扰是驱动森林自然更新的重要因子。 对具有丰富林下幼苗的受损程度较弱的常绿阔叶林 的管理, 可通过择伐部分先锋树种的方式改善林下 环境条件, 以促进林下幼苗的生长。对种子生产缺 乏和林下幼苗稀少的受损程度严重的森林, 可通过 直接在野外人为播种的方式促进种群幼苗的增补, 大、中林窗是播种的优先选择。此外, 应对幼苗向 幼树及幼树向成年个体的转化等过程进行长期地持 续监测和管理, 以更好地实现植物群落的持续发 展。

致谢 感谢重庆大学高祥阳、齐猛和周侠在实验中 给予的帮助; 感谢重庆市缙云山国家级自然保护区 管理局给予的支持; 感谢华东师范大学生态与环境 科学学院张健教授审改英文内容。

\section{参考文献}

Abe M, Miguchi H, Nakashizuka T (2001). An interactive effect of simultaneous death of dwarf bamboo, canopy gap, and predatory rodents on beech regeneration. Oecologia, 127, 281-286.

Bonal D, Burban B, Stahl C, Wagner F, Hérault B (2016). The response of tropical rainforests to drought-Lessons from recent research and future prospects. Annals of Forest Science, 73, 27-44.

Chanthorn W, Caughlin T (2013). The relative importance of fungal infection, conspecific density and environmental heterogeneity for seedling survival in a dominant topical tree. Biotropica, 45, 587-593.

Chen B, Da LJ, Song YC (2002). Seed germination and seedling growth of Castanopsis fargesii in evergreen broadleaved forest and in gaps. Journal of Tropical and Subtropical Botany, 10, 207-214. [陈波, 达良俊, 宋永昌 (2002). 常绿阔叶林内和林窗中栲树的种子萌发和幼苗 生长. 热带亚热带植物学报, 10, 207-214.]

Chen GP, Xian JR, Cao XW, Liu JQ, Yang YH, Lei W (2016). Effects of canopy gap on the survival dynamics of Abies faxoniana seedlings in a subalpine coniferous forest. Acta Ecologica Sinica, 36, 6475-6486. [陈国鹏, 鲜骏仁, 曹秀 文, 刘锦乾, 杨永红, 雷炜 (2016). 林窗对岷江冷杉幼 苗生存过程的影响. 生态学报, 36, 6475-6486.]

Chen SB, Song AQ, Li ZJ (2005). Research advance in response of forest seedling regeneration to light environmental heterogeneity. Chinese Journal of Applied Ecology, 16, 365-370. [陈圣宾, 宋爱琴, 李振基 (2005). 森林幼 苗更新对光环境异质性的响应研究进展. 应用生态学 报, 16, 365-370.]

Chmura DJ, Modrzyński J, Chmielarz P, Tjoelker MG (2017). Plasticity in seedling morphology, biomass allocation and physiology among ten temperate tree species in response to shade is related to shade tolerance and not leaf habit. Plant Biology, 19, 172-182.

Clark CJ, Poulsen JR, Levey DJ (2013). Roles of seed and establishment limitation in determining patterns of afrotropical tree recruitment. PLOS ONE, 8, e63330. DOI: 10.1371/journal.pone.0063330.

Comita LS, Hubbell SP (2009). Local neighborhood and species' shade tolerance influence survival in a diverse seedling bank. Ecology, 90, 328-334.

Ding SY, Lu XL (2007). Comparing ecophysiological characteristics of Castanopsis fargesii seedlings and saplings under different disturbance regimes: A case study of the Tiantong evergreen broad-leaved forest, Zhejiang Province of China. Acta Ecological Sinica, 27, 3892-3900. [丁圣 彦, 卢训令 (2007). 不同干扰背景下栲树(Castanopsis fargesii)幼苗幼树的生理生态特性比较——以浙江天童 常绿阔叶林为例. 生态学报, 27, 3892-3900.]

Dong H, Yang LX (2018). Effects of soil in larch plantations on the growth of Picea koraiensis and P. crassifolia seedlings. Chinese Journal of Applied Ecology, 29, 3199-3205. [董慧, 杨立学 (2018). 落叶松人工林土壤对红皮云杉 和青海云杉幼苗生长的影响. 应用生态学报, 29 , 3199-3205.]

Donohue K, Casas RRD, Burghardt L, Kovach K, Willis CG (2010). Germination, postgermination adaptation, and species

www.plant-ecology.com 
ecological ranges. Annual Review of Ecology \& Systematics, 41, 293-319.

Du YJ, Peng SJ, Xu GL, Huang ZL, Huang YJ (2007). Study of distance-dependence on Castanopsis Chinensis seed in coniferous and broad-leaved mixed forest of Dinghushan, China. Journal of Plant Ecology (Chinese Version), 31, 998-1006. [杜彦君, 彭闪江, 徐国良, 黄忠良, 黄玉佳 (2007). 鼎湖山针阔混交林锥栗种子距离制约研究. 植 物生态学报, 31, 998-1006.]

Engelbrecht BMJ, Dalling JW, Pearson TRH, Wolf RL, Gálvez DA, Koehler T, Tyree MT, Kursar TA (2006). Short dry spells in the wet season increase mortality of tropical pioneer seedlings. Oecologia, 148, 258-269.

Feng DL, Zhang LN, Huang ZH, Chen JH, Zhu XL (2013). Effects of gap size on the seed germination and seedling growth of Castanopsis carlesii and Castanopsis fargesii. Journal Northwest A\&F University (Nature Science), 41(6), 61-66. [冯大兰, 张丽楠, 黄仲华, 陈继红, 朱小 龙 (2013). 林窗大小对小叶栲和栲树种子萌发及幼苗 生长的影响. 西北农林科技大学学报(自然科学版), 41(6), 61-66.]

Figueroa JA, Lusk CH (2001). Germination requirements and seedling shade tolerance are not correlated in a Chilean temperate rain forest. New Phytologist, 152, 483-489.

Gao XY (2015). Castanopsis fargesii Decline and the Effect on Seedling Recruitment on Mt. Jinyun. Master degree dissertation, Chongqing University, Chongqing. [高祥阳 (2015). 缙云山栲树衰退及其幼苗更新. 硕士论文, 重 庆大学, 重庆.]

Gao XY, Huang L, Yang C, Yang YC, Yuan XZ (2016). Seed germination characteristics of the dominate tree Castanopsis fargesii in evergreen broadleaved forest on Mt. Jinyun. Journal of Chongqing Normal University (Nature Science), 33(2), 127-133. [高祥阳, 黄力, 杨超, 杨永川, 袁 兴中 (2016). 缙云山常绿阔叶林建群种栲树的种子萌 发特性. 重庆师范大学学报 (自然科学版), 33(2), 127-133.]

Gong HD, Yang GP, Lu ZY, Liu YH, Cao M (2011). Composition and spatio-temporal distribution of tree seedlings in an evergreen broad-leaved forest in the Ailao Mountain, Yunnan. Biodiversity Science, 19, 151-157. [巩合德, 杨 国平, 鲁志云, 刘玉洪, 曹敏 (2011). 哀牢山常绿榈叶 林乔木树种的幼苗组成及时空分布特征. 生物多样性, 19, 151-157.]

Gremer JR, Kimball S, Venable DL (2016). Within- and among-year germination in Sonoran Desert winter annuals: Bet hedging and predictive germination in a variable environment. Ecology Letters, 19, 1209-1218.

Hou ZJ, Zhao CZ, Li Y, Zhang Q, Ma XL (2014). Trade-off between height and branch numbers in Stellera chamaejasme on slopes of different aspects in a degraded alpine grassland. Chinese Journal of Plant Ecology, 38, 281-288.
[侯兆疆, 赵成章, 李钰, 张茜, 马小丽 (2014). 不同坡 向高寒退化草地狼毒株高和枝条数的权衡关系. 植物 生态学报, 38, 281-288.]

Huang L (2016). Seed Ecology of Subtropical Broad-leaved Evergreen Forest on Jinyun Mountain. Master degree dissertation, Chongqing University, Chongqing. [ 黄力 (2016). 缙云山常绿阔叶林种子生态学研究. 硕士论文, 重庆大学, 重庆.]

Jin Y, Russo SE, Yu M (2018). Effects of light and topography on regeneration and coexistence of evergreen and deciduous tree species in a Chinese subtropical forest. Journal of Ecology, 106, 1634-1645.

Larson JE, Sheley RL, Hardegree SP, Doescher PS, James JJ (2015). Seed and seedling traits affecting critical life stage transitions and recruitment outcomes in dryland grasses. Journal of Applied Ecology, 52, 199-209.

Larson JE, Sheley RL, Hardegree SP, Doescher PS, James JJ (2016). Do key dimensions of seed and seedling functional trait variation capture variation in recruitment probability? Oecologia, 181, 39-53.

Li QK, Ma KP (2003). Factors affecting establishment of Quercus liaotungensis Koidz. under mature mixed oak forest overstory and in shrubland. Forest Ecology \& Management, 176, 133-146.

Li YN, Li DS, Xu ZQ, Yu XF (2014). The process of emergence and height growth of Qucercus species in different provenances. Journal of Northwest Forestry University, 29(4)，139-144. [李亚男, 李东胜, 许中旗, 虞晓凡 (2014). 柇属不同种源树种的出苗及生长过程. 西北林 学院学报, 29(4), 139-144.]

Lu JM, Johnson DJ, Qiao XJ, Lu ZJ, Wang QG, Jiang MX (2015). Density dependence and habitat preference shape seedling survival in a subtropical forest in central China. Journal of Plant Ecology, 8, 568-577.

Ma LW, Zhang WH, Zhou JY, Xue YQ, Wu M, Ma C (2013). Effects of forest gap size on the growth of Quercus variabilis seedlings on north slopes of the Qinling Mountains. Scientia Silvae Sinicae, 49(12), 43-50. [马莉薇, 张文辉, 周建云, 薛瑶芹, 吴敏, 马闯 (2013). 秦岭北坡林窗大 小对栓皮栎实生幼苗生长发育的影响. 林业科学, 49(12), 43-50.]

Morales J, Squeo FA, Tracol Y, Armas C, Gutiérrez JR (2015). Resource economics and coordination among above- and below-ground functional traits of three dominant shrubs from the Chilean coastal desert. Journal of Plant Ecology, 8, 70-78.

Ou JD, Wu ZZ, Luo N (2016). Effects of forest gap size on the growth and form quality of Taxus wallichina var. mairei in Cunninghamia lanceolata forest. Chinese Journal of Applied Ecology, 27, 3098-3104. [欧建德, 吴志庄, 罗宁 (2016). 林窗大小对杉木林内南方红豆杉生长与形质的 影响. 应用生态学报, 27, 3098-3104.] 
Piiroinen T, Valtonen A, Roininen H (2017). The seed-to-seedling transition is limited by ground vegetation and vertebrate herbivores in a selectively logged rainforest. Forest Ecology \& Management, 384, 137-146.

Reddy KR, Brand D, Wijewardana C, Gao W (2017). Temperature effects on cotton seedling emergence, growth, and development. Agronomy Journal, 109, 1379-1387.

Song YC (2013). China Evergreen Broad-leaved Forest: Classification, Ecology, Conservation. Science Press, Beijing. [宋永昌 (2013). 中国常绿阔叶林: 分类·生态·保育. 科 学出版社, 北京.]

Tang JM, Zhai MP (2006). Morphological response and biomass allocation of Schima superba seedlings at different habitats in gap. Journal of Huazhong Agricultural University, 25, 559-563. [汤景明, 翟明普 (2006). 木荷幼苗在 林窗不同生境中的形态响应与生物量分配. 华中农业 大学学报, 25, 559-563.]

Tian D, Li P, Fang WJ, Xu J, Luo YK, Yan ZB, Zhu B, Wang JJ, Xu XN, Fang JY (2017). Growth responses of trees and understory plants to nitrogen fertilization in a subtropical forest in China. Biogeosciences, 14, 3461-3469.

Wang BC, Smith TB (2002). Closing the seed dispersal loop. Trends in Ecology \& Evolution, 17, 379-386.

Xing ZL, Zhang WH, Huang CZ, Yu SC, Zhou JY (2017). Effects of forest gap size on the aboveground architecture of Pinus tabulaeformis seedlings. Acta Botanica BorealiOccidentalia Sinica, 37, 578-585. [邢忠利, 张文辉, 黄 财智, 于世川, 周建云 (2017). 林窗大小对油松实生苗 地上部分构型的影响. 西北植物学报, 37, 578-585.]

Yan XF, Cao M (2008). Effects of forest gap on the seedling growth of a canopy tree species Pometia tomentosa in tropical rainforest of Xishuangbanna. Chinese Journal of Applied Ecology, 19, 238-244. [阊兴富, 曹敏 (2008). 林 窗对热带雨林冠层树种线毛番龙眼幼苗生长的影响. 应用生态学报, 19, 238-244.]

Yan XF, Wang JL, Zhou LB (2011). Effects of light intensity on Quercus Wutaishanica seed germination and seedling growth. Chinese Journal of Applied Ecology, 22, 1682-1688. [间兴富, 王建礼, 周立彪 (2011). 光照对 辽东柇种子萌发和幼苗生长的影响. 应用生态学报, 22 , 1682-1688.]

Yang YC, Huang L, Qian SH, Fukuda KJ (2015). Completing the life history of Castanopsis fargesii: Changes in the seed dispersal, seedling and sapling recruitment patterns. European Journal of Forest Research, 134, 1143-1154.

Zhang K, Baskin JM, Baskin CC, Yang X, Huang Z (2017). Effect of seed morph and light level on growth and reproduction of the amphicarpic plant Amphicarpaea edgeworthii (Fabaceae). Scientific Reports, 7, 39886.

Zheng YL, Sun WB, Zhou Y, Coombs D (2009). Variation in seed and seedling traits among natural populations of Trigonobalanus doichangensis (A. Camus) Forman (Fagaceae), a rare and endangered plant in southwest China. New Forests, 37, 285-294.

Zhou JH, Cheng XR, Yu MK, Zhuge JJ (2015). Responses of growth, leaf traits and biomass allocation of Phoebe sheareri seedlings to different habitats in forest gap. Journal of Northeast Forestry University, 43(12), 21-24, 50. [周俊宏, 成向荣, 虞木奎, 诸葛建军 (2015). 紫楠 幼苗生长、叶性状和生物量分配对林窗不同生境的响 应. 东北林业大学学报, 43(12), 21-24, 50.]

Zhu JJ, Gonda Y, Yu LZ, Li FQ, Yan QL, Sun YR (2012). Regeneration of a coastal pine (Pinus thunbergii Parl.) forest 11 years after thinning, Niigata, Japan. PLOS ONE, 7, e47593. DOI: 10.1371/journal.pone.0047593.

Zhu JJ, Matsuzaki T, Lee FQ, Gonda Y (2003). Effect of gap size created by thinning on seedling emergency, survival and establishment in a coastal pine forest. Forest Ecology \& Management, 182, 339-354. 